\title{
SUPER EFFICIENCY IN VECTOR OPTIMIZATION
}

\author{
J. M. BORWEIN AND D. ZHUANG
}

\begin{abstract}
We introduce a new concept of efficiency in vector optimization. This concept, super efficiency, is shown to have many desirable properties. In particular, we show that in reasonable settings the super efficient points of a set are norm-dense in the efficient frontier. We also provide a Chebyshev characterization of super efficient points for nonconvex sets and a scalarization theory when the underlying set is convex.
\end{abstract}

\section{INTRODUCTION}

Decision-making problems appearing in economics, management science and operations research require frequently that decision making be based on optimizing several criteria. Vector optimization has provided an organized constructive approach to these problems. Throughout this note, we consider minimization problems. Efficient decisions are those decisions not minorized by any others.

As observed by Kuhn and Tucker and later Geoffrion, a subset of an efficient decision set may not be satisfactorily characterized by a scalar minimization problem, so the concept of proper efficiency was introduced by Kuhn-Tucker, Geoffrion, and modified and formulated in a more general framework by Borwein, Benson, Henig, and Hartley among many other authors [Kuhn 1], [Benson 1], [Borwein 1, 2, 4, 6], [Geoffrion 1], [Henig 1], [Hartley 1] and the references therein. The motivation for introducing proper efficiency is that it enables one to eliminate certain anomalous efficient decisions and to prove the existence of equivalent scalar problems whose solutions produce at least most of the efficient decisions, namely the proper ones. It has been amply demonstrated that proper efficiency is a natural concept in vector optimization.

In this note, we introduce a new kind of proper efficiency, namely super efficiency. Super efficiency refines the notions of efficiency and other kinds of proper efficiency, and provides a concise (and equivalent) scalar characterization and duality results when the underlying decision problem is convex. We also

Received by the editors March 23, 1990 and, in revised form, March 6, 1991.

1980 Mathematics Subject Classification (1985 Revision). Primary 49A27, 90C31; Secondary 46A40, 52A07.

Key words and phrases. Vector optimization, efficiency, proper efficiency, super efficiency, Density Theorem, Chebyshev scalarization.

Research of the first author was partially supported by NSERC. Much of the material in this paper formed part of the second author's doctoral dissertation. 
study density results for super efficiency and a Chebyshev scalar characterization of super efficiency.

\section{Preliminaries}

For the sake of simplicity, we make the following assumptions (unless specifically stated otherwise). Throughout the paper, $X$ will always be an ordered real normed linear space and a subset $C$ of $X$ is always assumed to be nonempty. The partial ordering cone $S$ of $X$ is always assumed to be closed, convex $(S+S \subset S)$ and pointed, that is $S \cap-S=\{0\}$.

We say that the ordering cone $S$ of $X$ is generating if $X=S-S$. We associate a dual cone with $S$, denoted by $S^{+}$, in $X^{*}$ (the norm dual of $X$ ),

$$
S^{+}:=\left\{\phi \in X^{*} \mid \phi(s) \geq 0, \forall s \in S\right\} .
$$

Then $S^{+}$is a convex cone which is closed in $\sigma\left(X^{*}, X\right)$, the weak-star topology, and $S^{+}$is pointed if $S$ is generating.

Recall that an ordering cone is normal if $(B-S) \cap(S-B)$ is bounded, and so is a basic neighborhood. Here $B$ is the closed unit ball of the space $S$. Order intervals are defined by

$$
[a, b]_{s}:=\left\{x \mid a \leq_{S} x \leq_{S} b\right\} .
$$

Also $S^{+}$is a strict $b$-cone if for some $\varepsilon>0$,

$$
\phi \in X^{*}, \quad\|\phi\| \leq \varepsilon \Rightarrow \phi=\lambda_{1}-\lambda_{2}, \quad \lambda_{i} \in S^{+} \text {and }\left\|\lambda_{i}\right\| \leq 1, i=1,2 .
$$

In a Banach space, $S$ is normal if and only if $S^{+}$is generating and if and only if $S^{+}$is a strict $b$-cone [Peressini 1].

Recall that a base of a cone $S$ is a convex subset $\Theta$ of $S$ such that

$$
S=\bigcup\{\lambda \theta \mid \lambda \geq 0 \text { and } \theta \in \Theta\} \text { and } 0 \notin \operatorname{cl}(\Theta) .
$$

Of course, $S$ is pointed whenever $S$ has a base.

We say the ordering cone $S$ has the Daniell property if the infimum of each monotone decreasing net in $S$ exists and is also the topological limit of the net. In [Borwein 3] it is proved that $S$ is Daniell if

(i) $S$ has weakly compact intervals; or

(ii) if $S$ has a closed bounded complete base.

A vector lattice is an ordered vector space in which $\max \{a, b\}$ always exists. A normed lattice is a normed vector lattice in which the norm is absolute: $\|x\|=$ $\||x|\|$ and monotone: $0 \leq_{S} x \leq_{S} y$ implies that $\|x\| \leq\|y\|$. A Banach lattice is a Riesz lattice over a Banach space.

Recall also that the recession cone of a set $C$, denoted by $O^{+}(C)$, is the set of all $x$ such that

$$
c+\lambda x \in C \text { for all } \lambda>0 \text { and all } c \in C .
$$

The generalized (weak) recession cone of a set $C$, denoted by $R(C)\left[R_{\sigma}(C)\right]$, is the potentially larger set defined by

$$
\begin{gathered}
R(C):=\left\{\text { norm limits of } \lambda_{n} c_{n} \mid \lambda_{n} \rightarrow 0 \text { and } c_{n} \in C\right\} \\
{\left[R_{\sigma}(C):=\left\{\text { weak limits of } \lambda_{n} c_{n} \mid \lambda_{n} \rightarrow 0 \text { and } c_{n} \in C\right\}\right] .}
\end{gathered}
$$

The generalized (weak) recession cone of $C$ is the recession cone of $C$ when $C$ is closed and convex. Note that when $C$ is bounded, $R_{\sigma}(C)=\{0\}$. 
We shall frequently use the following notations:

(1) $\operatorname{cone}(A)$ denotes the cone generated by the set $A$, i.e.

$$
\operatorname{cone}(A):=\bigcup\{t A \mid t \geq 0\} ;
$$

while $\operatorname{cl}[\operatorname{cone}(A)]$ denotes the closure of $\operatorname{cone}(A)$.

(2) $S^{+i}$ denotes the set of all strictly positive linear functionals in $S^{+}$, that is

$$
S^{+i}:=\left\{f \in X^{*} \mid f(s)>0 \text {, for all } s \text { in } S \text { and } s \neq 0\right\} \text {. }
$$

It follows directly from the Hahn-Banach theorem that $S^{+i}$ is nonempty exactly when $S$ has a base.

(3) We use $\operatorname{int}(C)$ to denote the interior of the set $C$ and $C^{0}$ to denote the polar of $C$,

$$
C^{0}:=\left\{f \in X^{*} \mid f(c) \leq 1, \text { for all } c \text { in } C\right\} .
$$

(4) We denote the set of efficient points of $C$ with respect to the partial ordering cone $S$ by $E(C, S)$. Thus, $x \in E(C, S)$ if $(C-x) \cap-S=\{0\}$.

(5) $P E(C, S)$ denotes the set of all proper efficient points of $C$ in the sense of Borwein [Borwein 1]. That is, $x \in P E(C, S)$ if

$$
\operatorname{cl}[\operatorname{cone}(C-x)] \cap-S=\{0\} .
$$

(6) Let $S$ be an ordering cone in a normed linear space $X$ with a base $\Theta$, we can associate $S$ with another convex cone $S_{\varepsilon}(\Theta)$ defined by

$$
S_{\varepsilon}(\Theta):=\operatorname{cl}[\operatorname{cone}(\Theta+\varepsilon B)] \text {. }
$$

Here $B$ is the closed unit ball of $X$. We say $x \in C$ is Henig efficient, denoted by $x \in H E(C, S)$, [more properly, $x \in H E(C, \Theta)$ ] if

$$
\operatorname{cl}[\operatorname{cone}(C-x)] \cap-S_{\varepsilon}(\Theta)=\{0\} .
$$

$S_{\varepsilon}(\Theta)$, called the Henig Dilating Cone, plays an important role in our development. Some properties of $S_{\varepsilon}(\Theta)$ are summarized in the following theorem.

Theorem 1.1. Let $X$ be a normed linear space, $S \subset X$ a closed and convex cone with a closed base $\Theta$. Let $\delta:=\inf \{\|\theta\| \mid \theta \in \Theta\}$, then $\delta>0$. Define

$$
S_{\varepsilon}:=S_{\varepsilon}(\Theta):=\operatorname{cl}[\operatorname{cone}(\Theta+\varepsilon B)] \text {. }
$$

Then for any $\beta>0, S_{\varepsilon} \subset S_{\varepsilon+\beta}$. For $0 \leq \varepsilon<\delta$ we have

(1) $S_{\varepsilon}(\Theta)=\operatorname{cone}[\operatorname{cl}(\Theta+\varepsilon B)]$;

(2) $S_{\varepsilon}(\boldsymbol{\Theta})$ is pointed;

(3) $\cap\left\{S_{\varepsilon}(\Theta) \mid \varepsilon>0\right\}=S$;

(4) $S$ is normal if and only if $S_{\varepsilon}(\Theta)$ is normal;

(5) If, in addition, $X$ is complete and $\Theta$ is bounded then $S_{\varepsilon}(\Theta)$ is Daniell and normal;

(6) If $\Theta$ is weakly compact and $X$ is reflexive then $S_{\varepsilon}(\Theta)$ is locally weakly compact;

(7) If $X$ is reflexive and $S$ is normal, then $S_{\varepsilon}(\Theta)$ has weakly compact intervals and so is Daniell.

Proof. (1) Let

$$
\Theta_{\varepsilon}:=\operatorname{cl}(\Theta+\varepsilon B) .
$$


Since $S_{\varepsilon}(\boldsymbol{\Theta})$ is closed, $\operatorname{cl}\left[\operatorname{cone}\left(\Theta_{\varepsilon}\right)\right]=S_{\varepsilon}(\Theta)$. Let $d$ be in $\operatorname{cl}\left[\operatorname{cone}\left(\Theta_{\varepsilon}\right)\right]$. Then since $0 \notin \Theta_{\varepsilon}$ for $\varepsilon<\delta$,

$$
\operatorname{cl}\left[\operatorname{cone}\left(\Theta_{\varepsilon}\right)\right]=\operatorname{cone}\left(\Theta_{\varepsilon}\right) \cup R\left(\Theta_{\varepsilon}\right)
$$

where $R\left(\boldsymbol{\Theta}_{\varepsilon}\right):=\left\{\lim t_{\alpha} \theta_{\alpha}^{\varepsilon} \mid t_{\alpha} \rightarrow 0, t_{\alpha} \geq 0, \theta_{\alpha}^{\varepsilon} \in \Theta_{\varepsilon}\right\}$. Indeed, $x$ in cl[cone $\left.\left(\Theta_{\varepsilon}\right)\right]$ implies that $x$ is either in $\operatorname{cone}\left(\Theta_{\varepsilon}\right)$ or

$$
x=\lim t_{\alpha} \theta_{\alpha}^{\varepsilon} \text { for } t_{\alpha} \geq 0 \text { and } \theta_{\alpha}^{\varepsilon} \in \Theta_{\varepsilon} .
$$

Without loss of generality (taking subnets if necessary), we may assume that $t_{\alpha}$ converges. If $t_{\alpha}$ tends to infinity, then $t_{\alpha}^{-1}$ tends to zero, which implies that $\theta_{\alpha}^{\varepsilon}$ tends to zero. This is impossible as 0 is not in the closure of $\Theta$. Hence, $t_{\alpha}$ converges to $t$ for some $t<\infty$. If $t=0$ then $x$ is in $R\left(\Theta_{\varepsilon}\right)$ by definition. If $t \neq 0$ then as $\Theta_{\varepsilon}:=\operatorname{cl}(\Theta+\varepsilon B)$,

$$
\theta_{\alpha}^{\varepsilon} \rightarrow t^{-1} x \in \Theta_{\varepsilon} .
$$

Hence, $x \in \operatorname{cone}\left(\boldsymbol{\Theta}_{\varepsilon}\right)$. Therefore, (1.1) is verified. Note that it is easy to check that

$$
R\left(\boldsymbol{\Theta}_{\varepsilon}\right)=R(\Theta) \subset S \subset \operatorname{cone}\left(\Theta_{\varepsilon}\right)
$$

because $\Theta$ is a base for $S$ and $S$ is closed. Whence cone $\left(\Theta_{\varepsilon}\right)=S_{\varepsilon}(\Theta)$.

(2) By (1), $S_{\varepsilon}(\Theta)=\operatorname{cone}\left(\Theta_{\varepsilon}\right)$ has a closed base $\Theta_{\varepsilon}$, hence $S_{\varepsilon}(\Theta)$ is pointed.

(3) It is clear that $\bigcap_{\varepsilon>0} S_{\varepsilon} \supset S$. Let $d$ be in $\bigcap_{\varepsilon>0} S_{\varepsilon}$. We may assume that

$$
d=\lim t_{n}\left(\theta_{n}+(1 / n) b_{n}\right)
$$

with $\theta_{n} \in \boldsymbol{\Theta}, b_{n} \in B$ and $t_{n}>0$. Then

$$
\lim \left\|t_{n}^{-1} d-\Theta_{n}\right\|=\lim (1 / n)\left\|b_{n}\right\|=0 .
$$

Since $0 \in \Theta, t_{n}$ is bounded. Assume that $t_{n^{\prime}} \rightarrow \mu<\infty$. Note that since $\Theta$ is closed, we have $d=\mu \theta \in S$ for some $\theta \in \Theta$. Hence $d$ is in $S$.

(4) By [Peressini 1, p. 64; Borwein 6] the normality of $S$ is equivalent to the uniform boundedness of order intervals. Suppose that $S$ is normal, then order intervals of $S$ are uniformly norm-bounded. Let $0 \leq S_{\varepsilon} x \leq_{s_{\varepsilon}} a$ be given. Since

$$
x \in S_{\varepsilon}=\operatorname{cone}\left(\Theta_{\varepsilon}\right)=\operatorname{cone}[\operatorname{cl}(\Theta+\varepsilon B)] \subset \operatorname{cone}\left(\Theta+\varepsilon^{\prime} B\right)
$$

for any $\delta>\varepsilon^{\prime}>\varepsilon$, we may assume that

$$
x=t_{1}\left(\theta_{1}+\varepsilon^{\prime} b_{1}\right) ; \quad a-x=t_{2}\left(\theta_{2}+\varepsilon^{\prime} b_{2}\right)
$$

with $\theta_{i} \in \Theta, b_{i} \in B, t_{i}>0(i=1,2)$. Thus

$$
a=\left(t_{1}+t_{2}\right)\left(\theta+\varepsilon^{\prime} b\right)
$$

where $\theta=\left(t_{1} \theta_{1}+t_{2} \theta_{2}\right) /\left(t_{1}+t_{2}\right)$ and $b=\left(t_{1} b_{1}+t_{2} b_{2}\right) /\left(t_{1}+t_{2}\right)$. Define $s_{i}:=t_{i} \theta_{i}$ for $i=1,2$. Then

$$
s_{i} \leq s s_{1}+s_{2}=\left(t_{1}+t_{2}\right) \theta \text {. }
$$

By the normality of $S$, there exists a $k>0$, independent of $x$, such that

$$
\left\|s_{i}\right\| \leq k\left\|\left(t_{1}+t_{2}\right) \theta\right\|
$$

and so

$$
\begin{aligned}
\|x\| & \leq k\left\|\left(t_{1}+t_{2}\right) \theta\right\|+\varepsilon^{\prime}\left(t_{1}+t_{2}\right) \\
& \leq k\left\|\left(t_{1}+t_{2}\right) \theta+\left(t_{1}+t_{2}\right) \varepsilon^{\prime} b\right\|+k\left\|\left(t_{1}+t_{2}\right) \varepsilon^{\prime} b\right\|+\varepsilon^{\prime}\left(t_{1}+t_{2}\right) \\
& \leq k\|a\|+k\left(t_{1}+t_{2}\right) \varepsilon^{\prime}+\varepsilon^{\prime}\left(t_{1}+t_{2}\right) \leq k\|a\|+\varepsilon^{\prime}(1+k)\left(t_{1}+t_{2}\right) .
\end{aligned}
$$


Since $\Theta_{\varepsilon^{\prime}}$ is a base, the coefficient $t_{1}+t_{2}$ in (1.2) is uniformly bounded, say by $N\|a\|$ independent of $x$. Thus

$$
M(a):=k\|a\|+\varepsilon^{\prime}(1+k) N\|a\|
$$

is a constant independent of the choice of $x$. Hence, $[0, a]_{S_{\varepsilon}}$ is uniformly bounded in norm. Therefore $S_{\varepsilon}$ is normal. Conversely, if $S_{\varepsilon}$ is normal for some $\varepsilon>0$, then as $S_{\varepsilon} \supset S, S$ is normal.

(5) If $\Theta$ is bounded, then $S_{\varepsilon}=\operatorname{cone}[\operatorname{cl}(\Theta+\varepsilon B)]$ has a closed and bounded base. Since $X$ is complete, $S_{\varepsilon}$ is Daniell [Borwein 3]; $S_{\varepsilon}$ is also normal [Borwein 3].

(6) When $\Theta$ is weakly compact and $X$ is reflexive, $\theta+\varepsilon B$ is weakly compact. Hence $S_{\varepsilon}$ is locally weakly compact by a theorem of Klee [Klee 1].

(7) By (4), $S$ being normal implies that $S_{\varepsilon}$ is normal for $\varepsilon<\delta$. Thus order intervals of $S_{\varepsilon}$ are norm-bounded. They are also closed (because $S_{\varepsilon}$ is closed) and convex. Hence, intervals are weakly compact as $X$ is reflexive.

\section{SUPER EFFICIENCY}

In this section, we introduce a new efficiency concept, namely super efficiency. After defining the concept in a general setting we provide equivalent formulations of super efficiency for several important special cases. Existence results are then presented.

Definition 2.1. Let $X$ be a real normed linear space. We say that $x$ is a super efficient point of a nonempty subset $C$ of $X$ with respect to the ordering cone $S$, written $x \in S E(C, S)$, if there is a real number $M>0$ such that

$$
K \cap(B-S) \subset M B
$$

where $K:=\operatorname{cl}[\operatorname{cone}(C-x)]$ and $B$ is the closed unit ball of $X$.

Since $X$ is a normed linear space, $(2.1)$ is unchanged if $K$ is replaced by cone $(C-x)$. Thus, $(2.1)$ can be expressed explicitly in terms of the norm on $X$ :

for any $c$ in $C$ and $t>0$, whenever $t(c-x) \leq_{S} b$ for some $b \in B$,

$$
\|t(c-x)\| \leq M .
$$

This is equivalent to saying that for each $c$ in $C$, if $y$ is in $X$ and $c-x \leq_{s} y$, then

$$
\|c-x\| \leq M\|y\|
$$

with a uniform constant $M$ (depending only on $x$, not on $y$ or $c$ ).

Note that when $y=0,(2.2)$ becomes

$$
c-x \leq{ }_{S} 0 \Rightarrow\|c-x\|=0, \quad \text { for all } c \in C,
$$

which implies that super efficient points are efficient, i.e.

$$
S E(C, S) \subset E(C, S) \text {. }
$$

The relationships between super efficiency and other efficiencies will be explored in detail in the following sections. 
Super efficiency has a very simple description in a normed lattice. In this setting, $x \in S E(C, S)$ is equivalent to the existence of some $M$ with

$$
\|c-x\| \leq M\left\|(c-x)^{+}\right\|
$$

for each $c \in C$, where $(c-x)^{+}$is the positive part of $c-x$, see [Jameson 1].

To see this, we put $y:=(c-x)^{+}$in (2.2) and have (2.3) immediately. On the other hand, if $c-x \leq_{S} y$ for $y$ in $X$, then $(c-x)^{+} \leq_{S} y^{+}$. Hence

$$
\begin{aligned}
\|c-x\| & \leq M\left\|(c-x)^{+}\right\| \leq M\left\|(y)^{+}\right\| \quad(\text { as }\|\cdot\| \text { is monotone) } \\
& \leq M\||y|\|=M\|y\| \quad(\text { as }\|\cdot\| \text { is absolute })
\end{aligned}
$$

which is exactly $(2.3)$.

When the set $C$ is convex, our definition of super efficiency has a concise dual form. Indeed, we prove in the following proposition that (2.1) is equivalent to

$$
X^{*}=K^{+}-S^{+}=(C-x)^{+}-S^{+} .
$$

Proposition 2.2. Let $X$ be a Banach space, $C \subset X$ a convex subset and $S$ a closed cone ( $S$ as always is convex). Then (2.1) is equivalent to (2.4).

Proof. When $C$ is convex, $K:=\operatorname{cl}[\operatorname{cone}(C-x)]$ is also convex. Hence

$$
H(x):= \begin{cases}x+S, & x \in K, \\ \varnothing, & x \notin K,\end{cases}
$$

is a closed convex process. It follows from direct calculations that for any $y$ in $X, H^{-1}(y)=K \cap(y-S)$ and the adjoint process [Borwein 5] is

$$
H^{*}\left(y^{*}\right)= \begin{cases}y^{*}-K^{+}, & y^{*} \in S^{+}, \\ \varnothing, & y^{*} \notin S^{+} .\end{cases}
$$

By definition, $x$ is in $S E(C, S)$ if and only if $H^{-1}$ is bounded which is equivalent, by the dual version of Corollary 2.1 in [Borwein 5] (as $H$ is closed), to $H^{*}$ being open at 0 , which in turn is equivalent to $H^{*}$ being surjective:

$$
H^{*}\left(K^{+}\right)=K^{+}-S^{+}=X^{*} \text {. }
$$

This is (2.4).

The following corollary gives a characterization of super efficiency by scalar optimization.

Corollary 2.3. Let $S$ be a normal cone in a normed space $X$. For any convex set $C$ of $X, x_{0}$ is in $S E(C, S)$ if and only if for any $\psi$ in $S^{+}$(or in $\left.X^{*}\right)$, one can find $\phi$ in $S^{+}$such that $\phi \geq_{S^{+}} \psi$ and

$$
\inf _{x \in C} \phi(x)=\phi\left(x_{0}\right) \text {. }
$$

Proof. Note that $S$ is normal in a normed space if and only if $S^{+}$is generating [Peressini 1, pp. 72 and 76] while (2.5) is equivalent to $S^{+} \subset\left(C-x_{0}\right)^{+}-S^{+}$. (Moreover, (2.5) implies that $X^{*}=S^{+}-S^{+}$.)

Now we present our first existence result for super efficiency. 
Proposition 2.4. Let $X$ be a normed space, $S \subset X$ an ordering cone with a bounded base and $C$ a weakly compact subset of $X$. Then $S E(C, S) \neq \varnothing$.

Proof. Since $S$ has a bounded base $\Theta$,

$$
\operatorname{norm}-\operatorname{int}\left(S^{+}\right) \neq \varnothing .
$$

[Jameson 1, p. 122]. Let $\psi$ be in $\operatorname{int}\left(S^{+}\right)$, then $\psi+\varepsilon B^{*} \subset S^{+}$for some $\varepsilon>0$, where $B^{*}$ is the closed unit ball in $X^{*}$. Thus for any $\lambda \in X^{*}$, we can always find $n>0$ so that $\lambda \leq S_{S^{+}} n \psi$. Moreover, since $C$ is weakly compact, there is $x_{0}$ in $C$ such that

$$
\min _{x \in C} \psi(x)=\psi\left(x_{0}\right) .
$$

In other words, for all $c$ in $C$ we always have $\psi\left(c-x_{0}\right) \geq 0$. Note also that since $S$ has a bounded base, it is normal [Borwein 3]. The fact that $x_{0}$ is in $S E(C, S)$ now follows from Corollary 2.3.

The following proposition and its corollary describes super efficiency in terms of the Henig dilating cone $S_{\varepsilon}$.

Proposition 2.5. Let $X$ be a normed linear space, $S \subset X$ a closed ordering cone, $C \subset X$ a nonempty subset and $x_{0} \in C$. Define $K:=\operatorname{cl}\left[\operatorname{cone}\left(C-x_{0}\right)\right]$ as before. Suppose that $S$ has a bounded base $\Theta$ and let $\delta:=\inf \{\|\theta\| \mid \theta \in \Theta\}$. Suppose also that for some $0<\varepsilon<\delta$,

$$
K \cap-S_{\varepsilon}=\{0\}
$$

Then $x_{0} \in S E(C, S)$.

Proof. Suppose that $x_{0}$ is not in $S E(C, S)$. Then we can select a sequence $\left\{y_{n}\right\}$ in $K \cap(B-S)$ with $\left\|y_{n}\right\|$ approaching to infinity, where $B$ is again the closed unit ball of $X$. Since $y_{n}$ is in $B-S$ and $S$ has a base $\Theta, y_{n}$ can be written as

$$
y_{n}=b_{n}-\lambda_{n} \theta_{n} \quad \text { and } \quad y_{n} \in K
$$

with $\theta_{n} \in \Theta,\left\|b_{n}\right\| \leq 1$, and $\lambda_{n}>0, n=1,2, \ldots$.

Since $\Theta$ is bounded, we may assume that $\left\|\theta_{n}\right\| \leq M, n=1,2, \ldots$. Now

$$
\left\|y_{n}\right\|-1 \leq\left\|y_{n}-b_{n}\right\| \leq \lambda_{n} M \text {. }
$$

Hence $\lambda_{n}$ tends to infinity. Therefore,

$$
0 \neq \lambda_{n}^{-1} y_{n}=\lambda_{n}^{-1} b_{n}-\theta_{n} \in K \cap(\varepsilon B-\Theta) \subset K \cap-S_{\varepsilon}
$$

for $n$ sufficiently large, which contradicts (2.6).

Corollary 2.6. Let $X$ be a normed space and suppose the ordering cone $S$ have a closed base. Let $\delta:=\inf \{\|\theta\| \mid \theta \in \Theta\}$ and let $S_{\varepsilon}:=S_{\varepsilon}(\Theta)$. Then for any set $C$ in $X$ the following are equivalent:

(1) $x_{0} \in E\left(C, S_{\varepsilon}\right)$ for some $0<\varepsilon<\delta$;

(2) $x_{0} \in P E\left(C, S_{\varepsilon}\right)$ for some $0<\varepsilon<\delta$.

If, in addition, $\boldsymbol{\theta}$ is a bounded base, then the above are equivalent to

(3) $x_{0} \in S E(C, S)$.

Proof. From the definitions

$$
P E\left(C, S_{\varepsilon}\right) \subset E\left(C, S_{\varepsilon}\right)
$$


is clear for any $\varepsilon>0$. On the other hand, $x_{0}$ is in $E\left(C, S_{\varepsilon}\right)$ if and only if

$$
\text { cone }\left(C-x_{0}\right) \cap-S_{\varepsilon}=\{0\} \text {. }
$$

By Theorem 1.1(1), for $0<\varepsilon<\delta, \operatorname{cl}(\varepsilon B-\Theta)$ is a base and $-S_{\varepsilon}=$ $\operatorname{cone}[\operatorname{cl}(\varepsilon B-\Theta)]$. Thus, for any $0<\varepsilon^{\prime}<\varepsilon$, cone $\left(C-x_{0}\right) \cap\left(\varepsilon^{\prime} B-\Theta\right)=\varnothing$. This implies that

$$
\text { cone }\left(C-x_{0}\right) \cap\left[\left(2^{-1} \varepsilon^{\prime} B-\Theta\right)+2^{-1} \varepsilon^{\prime} \operatorname{int}(B)\right]=\varnothing .
$$

Noting that the second set is open, we have,

$$
\operatorname{cl}\left[\operatorname{cone}\left(C-x_{0}\right)\right] \cap \operatorname{cl}\left(2^{-1} \varepsilon^{\prime} B-\Theta\right)=\varnothing .
$$

Therefore, by Theorem 1.1(1) again,

$$
\operatorname{cl}\left[\operatorname{cone}\left(C-x_{0}\right)\right] \cap \operatorname{cone}\left[\operatorname{cl}\left(2^{-1} \varepsilon^{\prime} B-\Theta\right)\right]=\operatorname{cl}\left[\operatorname{cone}\left(C-x_{0}\right)\right] \cap-S_{\varepsilon^{\prime \prime}}=\{0\}
$$

for $\varepsilon^{\prime \prime}:=2^{-1} \varepsilon^{\prime}$. This proves that $x_{0}$ is in $P E\left(C, S_{\varepsilon^{\prime \prime}}\right)$. Hence (1) implies (2).

Now let us assume that $S$ has a bounded base. Then $(2) \Rightarrow(3)$ follows from Proposition 2.5. Conversely, suppose $x_{0}$ is in $S E(C, S)$ while $K \cap-S_{\varepsilon}=\{0\}$ fails for all $\varepsilon>0$. Then for $n=1,2, \ldots$,

$$
K \cap(1 / n B-\Theta) \neq \varnothing \text {. }
$$

Take $k_{n}$ in $K \cap((1 / n) B-\Theta)$. Note that for $n$ sufficiently large,

$$
\left\|k_{n}\right\| \geq \delta-1 / n>0 \text {. }
$$

Now $n k_{n}$ is in $K \cap(B-S)$ with $\left\|n k_{n}\right\| \rightarrow \infty$. This contradicts the assumption that $x_{0}$ is in $S E(C, S)$.

Our next theorem says that every bounded closed set in a Banach space has super efficient points provided the ordering cone has a bounded base.

Theorem 2.7. Let $X$ be a Banach space and let $S$ have a closed bounded base $\Theta$. Then every bounded closed set $C$ possesses super efficient points.

Proof. Let $\delta:=\inf \{\|\theta\| \mid \theta \in \Theta\}$. Let $S_{\varepsilon}(\Theta)$ be the Henig cone for $0<\varepsilon<\delta$. Then $S_{\varepsilon}$ has a closed bounded base and $\operatorname{int}\left(S_{\varepsilon}\right)$ is nonempty. Thus $S_{\varepsilon}$ is Daniell [by Theorem 1.1(5)] and $C$ is bounded below in $S_{\varepsilon}$. By Theorem 2.1 in [Borwein 4], $E\left(C, S_{\varepsilon}\right)$ is nonempty. Hence, $S E(C, S)$ is nonempty by Corollary 2.6.

Example 2.8. (a) Let $X=l_{1}(\mathbb{N})$ and $S=l_{1^{+}}(\mathbb{N})$ (the natural ordering cone). Then $S$ has a bounded base. Let $C$ be the closed unit ball of $X$, i.e.,

$$
C=\left\{x \in l_{1}(\mathbb{N}) \mid\|x\|_{1} \leq 1\right\} .
$$

Let $\phi=(1,1, \ldots)$ be in $l_{\infty}(\mathbb{N})=\left[l_{1}(\mathbb{N})\right]^{*}$. Then

$$
\inf \{\phi(x) \mid x \in C\}=-1 \text {. }
$$

Note that $\phi$ attains its minima on $T:=\left\{x \in C \mid\|x\|=1\right.$ and $\left.x \leq_{s} 0\right\}$. Note also that for any $\lambda$ in $l_{\infty}(\mathbb{N})$ we can always find an integer $n>0$ so that $\lambda \leq_{S^{+}} n \phi$. Thus $S E(C, S)$ contains $T$ by Corollary 2.3. On the other hand, if $x$ is in $C \backslash T$, then $x$ is not even efficient. Thus, $S E(C, S)=T$.

(b) If we let

$$
C_{1}=\left\{x \in l_{1}(\mathbb{N}) \mid \sum x_{n} / 2^{n}=0\right\}
$$


Then $S E\left(C_{1}, S\right)=\varnothing$ while $P E\left(C_{1}, S\right)=C_{1}$. Indeed,

$$
C_{1}-x=C_{1}=\operatorname{cl}\left[\operatorname{cone}\left(C_{1}-x\right)\right]=: K
$$

for all $x$ in $C_{1}$ (since $C_{1}$ is a closed subspace) and $K \cap-S=\{0\}$. Hence $P E\left(C_{1}, S\right)=C_{1}$. But, since $\left(1 / 2^{n}\right) \notin \operatorname{int}\left(S^{+}\right)$,

$$
C_{1}^{+}-S^{+}=R\left(2^{-n}\right)-S^{+} \neq l_{\infty}(\mathbb{N}),
$$

where, $\left(C_{1}\right)^{+}=R\left(2^{-n}\right)$ is the set of all scalar multiples of $\left(2^{-n}\right)$ as $C_{1}$ is the kernel of $\left(2^{-n}\right)$. In particular, $(1,1,1, \ldots)$ is not in $\left(C_{1}\right)^{+}-S^{+}$. Therefore, $S E\left(C_{1}, S\right)=\varnothing$ by Proposition 2.2.

Note that $C$ is bounded while $C_{1}$ is not. So the example shows that the boundedness of $C$ is needed in Theorem 2.7. The following proposition gives a scalar characterization of super efficient points in an important case.

Proposition 2.9. Let $X$ be a normed space. If $S$ has a closed bounded base $\Theta$ and $C$ is convex then $x$ is in $S E(C, S)$ if and only if there is $\phi$ in the norm-interior of $S^{+}$such that $\phi(C-x) \geq 0$.

Proof. If $x$ is in $S E(C, S)$ then

$$
(C-x)^{+}-S^{+}=X^{*}
$$

(by Proposition 2.2). Since $\Theta$ has a bounded base, int $\left(S^{+}\right) \neq \varnothing$ [Jameson 1]. Therefore,

$$
(C-x)^{+}-\operatorname{int}\left(S^{+}\right)=X^{*},
$$

since $(C-x)^{+}-\operatorname{int}\left(S^{+}\right)$is a convex set with nonempty interior whose closure is $X^{*}$. (It then must be equal to $X^{*}$ otherwise, it is contained in a closed half-space.) This implies that

$$
0 \in(C-x)^{+}-\operatorname{int}\left(S^{+}\right) .
$$

So there is $\phi$ in $X^{*}$ such that

$$
\phi \in(C-x)^{+} \cap \operatorname{int}\left(S^{+}\right) .
$$

Conversely,

$$
\phi \in(C-x)^{+} \cap \operatorname{int}\left(S^{+}\right)
$$

implies that

$$
0 \in(C-x)^{+}-\operatorname{int}\left(S^{+}\right) \subset \operatorname{int}\left[(C-x)^{+}-S^{+}\right],
$$

which gives $X^{*}=K^{+}-S^{+}$and hence $x$ is in $S E(C, S)$.

\section{SUPER EFFICIENCY VIA OTHER EFFICIENCIES}

In this section, we shall consider in detail the relationships between super efficiency and other efficiencies such as Borwein efficiency, Henig efficiency, and Hartley efficiency. By exploring such relationships, we show that super efficiency is indeed a central kind of proper efficiency.

Proposition 3.1. Let $X$ be a normed space, $S$ an ordering cone and $C$ a subset of $X$. Then,

$$
S E(C, S) \subset P E(C, S) .
$$


Proof. Let $x$ be in $S E(C, S)$. For each $d$ in $\operatorname{cl}[\operatorname{cone}(C-x)] \cap-S$, we assume that $d=\lim d_{n}$ with $d_{n}$ in $\operatorname{cone}(C-x)$. Let $d_{n}=t_{n}\left(c_{n}-x\right)$ with $c_{n}$ in $C$, $t_{n}>0$. For all $n$, we have

$$
d_{n} \in-S+\left\|d_{n}-d\right\| B .
$$

Thus for some $b_{n}$ in $B$,

$$
c_{n}-x \leq_{S} t_{n}^{-1}\left\|d_{n}-d\right\| b_{n} .
$$

As $x$ is in $S E(C, S)$, there exists $M>0$ with $\left\|c_{n}-x\right\| \leq M t_{n}^{-1}\left\|d_{n}-d\right\|$, or

$$
\left\|d_{n}\right\| \leq M\left\|d_{n}-d\right\|,
$$

which implies that $\|d\| \leq 0$. Therefore

$$
\operatorname{cl}[\text { cone }(C-x)] \cap-S=\{0\} .
$$

Our next proposition indicates the important fact that in a finite dimensional space, super efficiency is the same as proper efficiency.

Proposition 3.2. Let $X$ be a normed linear space with $S$ a closed and convex ordering cone. Let $C$ be a closed subset of $X$ and lie in a finite dimensional subspace of $X$. Then

$$
S E(C, S)=P E(C, S) .
$$

Proof. It suffices to prove that

$$
P E(C, S) \subset S E(C, S) .
$$

Let $x$ be in $P E(C, S)$ which is contained in $E(C, S)$. If $x$ is not in $S E(C, S)$, then for $n=1,2, \ldots$ we can select $c_{n}$ in $C, h_{n}$ in $X$ with $c_{n} \leq_{s} x+h_{n}$ and

$$
\left\|c_{n}-x\right\|>2^{n}\left\|h_{n}\right\| \text {. }
$$

If $\left\|h_{n}\right\|=0$, then $c_{n} \leq_{S} x$. This violates the fact that $x$ is in $E(C, S)$. So $\left\|h_{n}\right\| \neq 0$. Let $d_{n}:=\left(c_{n}-x\right) /\left\|c_{n}-x\right\|$, then we have

$$
d_{n} \leq_{S} h_{n} /\left\|c_{n}-x\right\| \text {. }
$$

As $\left\{d_{n}\right\}$ is in a finite dimensional space and is bounded, we may assume (by extracting a subsequence if necessary) that $d_{n} \rightarrow d_{0}$. Then

$$
d_{0} \in \operatorname{cl}[\operatorname{cone}(C-x)]=K,
$$

and $d_{0}=\lim d_{n} \leq S \lim h_{n} /\left\|c_{n}-x\right\|=0$ by (3.4) and (3.3). Since $\left\|d_{n}\right\|=1$ for all $n, d_{0} \neq 0$. This contradicts our assumption that $x \in P E(C, S)$.

The next two propositions show that every super efficient point is a Henig efficient point; when the base of the cone is bounded, the two efficiencies are the same.

Proposition 3.3. Let $X$ be a normed linear space with an ordering cone $S$. If $S$ has a closed base $\Theta$, then

$$
S E(C, S) \subset H E(C, \Theta) \text {. }
$$

Proof. Let $\delta:=\inf \{\|\theta\| \mid \theta \in \Theta\}$. Then $\delta>0$. Suppose $x$ is in $\operatorname{SE}(C, S)$, then there exists a constant $M>0$ such that for each $c$ in $C$,

$$
c-x \leq_{s} y \Rightarrow\|c-x\| \leq M\|y\| \text { for all } y \text { in } X \text {. }
$$


Let $\varepsilon$ be chosen so that $\varepsilon<\delta /(1+M)$. Suppose there is $z$ such that

$$
z \in \operatorname{cone}(C-x) \cap(\varepsilon B-\Theta) \text {, }
$$

it then follows that

$$
z=\lambda(c-x)=\varepsilon b-\theta \leq \leq_{s} \varepsilon b
$$

for $b$ in $B$ and $\theta$ in $\Theta$ and $\lambda>0$. Thus, $\lambda^{-1}\|z\| \leq \lambda^{-1} M \varepsilon$ because $x$ is in $S E(C, S)$. Hence,

$$
\lambda^{-1}(\delta-\varepsilon) \leq \lambda^{-1}\|\theta-\varepsilon b\|=\lambda^{-1}\|z\| \leq \lambda^{-1} M \varepsilon
$$

and $\delta /(M+1) \leq \varepsilon$. This is a contradiction. Therefore,

$$
\operatorname{cone}(C-x) \cap(\varepsilon B-\Theta)=\varnothing \text {. }
$$

This implies that

$$
\text { cone }(C-x) \cap \operatorname{cone}(\varepsilon B-\Theta)=\{0\} \text {. }
$$

Thus, as cone $\left[\operatorname{cl}\left(\varepsilon^{\prime} B-\Theta\right)\right] \subset \operatorname{cone}(\varepsilon B-\Theta)$ for some $0<\varepsilon^{\prime}<\varepsilon$, one has

$$
\text { cone }(C-x) \cap-S_{\varepsilon^{\prime}}=\{0\} \text {. }
$$

Thus $x \in E\left(C, S_{\varepsilon^{\prime}}\right) \subset H E(C, \Theta)$.

Proposition 3.4. If $X$ is a normed linear space and $S \subset X$ is an ordering cone with a norm-bounded base $\Theta$, then

$$
H E(C, \Theta)=S E(C, S) \text {. }
$$

Proof. If $x \in H E(C, \Theta)$, then $x \in E\left(C, S_{\varepsilon}\right)$ for small $\varepsilon>0$. Thus

$$
\operatorname{cone}(C-x) \cap(\varepsilon B-\Theta)=\varnothing \text {. }
$$

Take any $y$ in $X$ with $c-x \leq_{S} y$, then we may assume that

$$
c-x=y-\lambda \theta
$$

for some $\theta$ in $\theta$ and $\lambda \geq 0$. If $\lambda=0$, then $\|c-x\|=\|y\|$. If $\lambda>0$,

$$
\lambda^{-1}(c-x)=\left(\lambda^{-1} y-\theta\right) \text {. }
$$

Now by (3.6) $\left\|\lambda^{-1} y\right\| \geq \varepsilon$, or

$$
\lambda /\|y\| \leq \varepsilon^{-1} .
$$

If we define $m:=\sup \{\|\theta\| \mid \theta \in \Theta\} \quad(m$ is finite as $\Theta$ is bounded) then

$$
\begin{aligned}
\|c-x\| & \leq\|y\|+\lambda \sup \|\theta\| \\
& \leq\|y\|+\lambda m=\|y\|(1+\lambda m /\|y\|) \\
& \leq\|y\|\left(1+\varepsilon^{-1} m\right)=M\|y\|, \quad[\text { by (3.7)] }
\end{aligned}
$$

where $M:=1+\varepsilon^{-1} m$. Therefore we have $\|c-x\| \leq M\|y\|$ whenever $c-x \leq s$ $y$. In other words, $x$ is in $S E(C, S)$.

Let us recall Hartley's notion of proper efficiency which is extended in [Borwein 2]: a point $x$ of a set $C$ in a normed space $X$ with an ordering cone $S$ is a Hartley efficient point, denoted by $x \in H P E(C, S)$, if there exists a constant $M>0$ such that whenever there is $\phi \in S^{+}$with $\phi(c-x)<0$ for some $c$ in $C$ one can find $\psi \in S^{+}$with

$$
\phi(c-x) /\|\phi\| \geq-M \psi(c-x) /\|\psi\| .
$$

In our next two propositions, we compare Hartley efficiency with super efficiency. Again, super efficient points are Hartley efficient. The two efficiencies coincide when the ordering cone is normal. 
Proposition 3.5. In a normed linear space setting we always have

$$
S E(C, S) \subset H P E(C, S) \text {. }
$$

Proof. Suppose that $x$ is in $S E(C, S)$. Then there is $M>0$ such that for each $c$ in $C$ and $h$ in $X$,

$$
c \leq s_{S} x+h \text { implies }\|c-x\| \leq M\|h\| .
$$

If $x$ is not in $\operatorname{HPE}(C, S)$ and $\varepsilon>0$, the definition of $\operatorname{HPE}(C, S)$ fails for $M+\varepsilon$. Then there exist $s_{0}^{+} \in S^{+}$with $\left\|s_{0}^{+}\right\|=1$ and $x \neq c_{0} \in C$ such that $s_{0}^{+}\left(c_{0}-x\right)<0$; and for all $s^{+}$in $S^{+},\left\|s^{+}\right\|=1$,

$$
s_{0}^{+}\left(x-c_{0}\right)>(M+\varepsilon) s^{+}\left(c_{0}-x\right) \text {. }
$$

This implies that for all $s^{+}$in $S^{+} \cap \partial B^{*}\left(\partial B^{*}\right.$ is the unit sphere in $\left.X^{*}\right)$,

$$
\left\|x-c_{0}\right\| \geq(M+\varepsilon) s^{+}\left(c_{0}-x\right) \text {. }
$$

Thus for all $s^{+}$in $S^{+} \cap B^{*}$

$$
s^{+}\left(c_{0}-x\right) /\left\|c_{0}-x\right\| \leq 1 /(M+\varepsilon) .
$$

Therefore, a standard polarity argument shows

$$
\left(c_{0}-x\right) /\left\|c_{0}-x\right\| \in(M+\varepsilon)^{-1}\left(S^{+} \cap B^{*}\right)^{0}=(M+\varepsilon)^{-1} \operatorname{cl}(B-S) .
$$

Hence, $c_{0}-x \in\left\|c_{0}-x\right\|(M+\varepsilon / 2)^{-1}(B-S)$, and as $x \in S E(C, S)$,

$$
\left\|c_{0}-x\right\| \leq[M /(M+\varepsilon / 2)]\left\|c_{0}-x\right\|<\left\|c_{0}-x\right\| \text {. }
$$

This is a contradiction.

Proposition 3.6. Let $X$ be a normed linear space with a normal ordering cone $S$. Then for any subset $C$ of $X, H P E(C, S)=S E(C, S)$.

Proof. Let $x$ be in $\operatorname{HPE}(C, S)$. Set

$$
\|y\|_{S}:=\sup \left\{\left|s^{+}(y)\right|: s^{+} \in S^{+},\left\|s^{+}\right\|=1\right\} \leq\|y\| .
$$

By the normality of $S$, there is a constant $M_{0}>0$ such that for all $y$ in $X$, $\|y\| \leq M_{0}\|y\|_{S}$, (since $S^{+}$is a strict $b$-cone, [Peressini 1, p. 76]). Suppose that $c-x \leq s y$, then for each $s^{+}$in $S^{+} \cap \partial B^{*}, s^{+}(c-x) \leq s^{+}(y)$. By the definition of $\|\cdot\|_{S}$, there is, for each $\varepsilon>0$, an $s_{1}^{+}$in $S^{+} \cap \partial B^{*}$ such that $\|c-x\|_{s} \leq\left|s_{1}^{+}(c-x)\right|(1+\varepsilon)$. If $s_{1}^{+}(c-x)>0$, then

$$
(1+\varepsilon)^{-1}\|c-x\|_{S} \leq s_{1}^{+}(c-x) \leq s_{1}^{+}(y) \leq\|y\| .
$$

If $s_{1}^{+}(c-x)<0$, then as $x$ is in $\operatorname{HPE}(C, S)$, there exists $s_{2}^{+}$in $S^{+} \cap \partial B^{*}$ and $M_{1}>0$ such that,

$$
s_{1}^{+}(x-c) \leq M_{1} s_{2}^{+}(c-x) \leq M_{1} s_{2}^{+}(y) \leq M_{1}\|y\|_{S} \leq M_{1}\|y\| .
$$

Thus in both cases, we have for all $\varepsilon>0,\|c-x\|_{S} \leq(1+\varepsilon) M_{2}\|y\|$, with $M_{2}:=\max \left\{1, M_{1}\right\}$. Therefore, $\|c-x\|_{S} \leq M_{2}\|y\|$. Finally, we have $\|c-x\| \leq$ $M_{0}\|c-x\|_{S} \leq M\|y\|$, where $M=M_{0} M_{2}$. Hence $x$ belongs to $S E(C, S)$.

When $X$ is $\mathbb{R}^{n}$ and $S$ is the nonnegative orthant $\mathbb{R}_{+}^{n}$, the definition of Hartley efficiency (equivalently super efficiency) coincides with Geoffrion's definition of proper efficiency [Geoffrion 1]. This emphasizes again that super efficient points are indeed the right subclass of efficient points. This is also the point of the following proposition. 
Proposition 3.7. Let $X$ be a normed linear space whose ordering cone $S$ has a weakly compact base. Then for any subset $C$ of $X, P E(C, S)=S E(C, S)$.

Proof. It suffices to show that any proper efficient point is super efficient. Let $x$ be in $P E(C, S)$. If we define $K:=\operatorname{cl}[\operatorname{cone}(C-x)]$ as usual, then $K \cap-S=$ $\{0\}$. We claim that there is an $\varepsilon>0$ such that

$$
K \cap(\varepsilon B-S) \subset(1 / \varepsilon) B \text {. }
$$

Indeed, if (3.8) is not true, then we can $y_{n} \in((1 / n) B-S) \cap K$ such that $\left\|y_{n}\right\|>n$. Assume that $y_{n}=b_{n} / n-s_{n}$ with $b_{n}$ in $B$ and $s_{n}$ in $S$. Now consider $s_{n} /\left\|y_{n}\right\|$,

$$
\left\|s_{n}\right\| /\left\|y_{n}\right\| \leq 1+1 /\left(n\left\|y_{n}\right\|\right)
$$

so $\left\{s_{n} /\left\|y_{n}\right\|\right\}$ is bounded. As $S$ is locally weakly compact, it has a weakly compact base $\boldsymbol{\Theta}$ [Klee 1], we may assume that $s_{n} /\left\|y_{n}\right\|=\lambda_{n} \theta_{n}$ where $\lambda_{n}>0$ and bounded. Now $\theta_{n}=\lambda_{n}^{-1}\left[b_{n}\left(n\left\|y_{n}\right\|\right)^{-1}-y_{n}\left\|y_{n}\right\|^{-1}\right]$ converges weakly to some $0 \neq \theta_{0}$ in $\Theta$. Hence $y_{n}\left\|y_{n}\right\|^{-1}$ converges weakly to some $0 \neq-s_{0}$ in $K \cap-S$. This contradicts our assumption that $x$ is in $\operatorname{PE}(C, S)$. Therefore (3.8) is verified. And so is $\operatorname{PE}(C, S) \subset S E(C, S)$.

\section{DENSITY THEOREMS FOR SUPER EFFICIENCY}

We have seen that in general the set of super efficient points is strictly contained in that of the efficient points. In this section we provide the answer to the question of "how many" efficient points are super efficient. First we need another definition.

Definition 4.1. Let $C$ be a subset of a normed linear space $X$ with a closed ordering cone $S$ and let $B$ be the closed unit ball in $X$. We say that $C$ is $S$-lower bounded if there is some constant $M>0$ such that

$$
C \subset M B+S \text {. }
$$

Note that if $C$ is $S$-lower bounded then

$$
R_{\sigma}(C) \subset S \text {. }
$$

(See $\S 1$ for the definition of $R_{\sigma}(C)$.) Indeed, let $d \in R_{\sigma}(C)$. By the definition of $R_{\sigma}(C)$, there exist $t_{n} \rightarrow 0$ and $c_{n} \in C$ such that $t_{n} c_{n}$ tends to $d$ weakly. As $C$ is $S$-lower bounded there are $b_{n} \in B$ and $M>0$ such that $c_{n}-M b_{n} \in S$. Now, $t_{n} c_{n}-t_{n} M b_{n} \in S$ and $t_{n} M b_{n} \rightarrow 0$ implies that $d$ is in $S$ as $S$ is closed and convex, hence weakly closed. Hence (4.2) holds. As $S$ is pointed we also see that $R_{\sigma}(C) \cap-S=\{0\}$.

Note that (4.1) holds if either $C$ is bounded topologically or $C$ has an $S$-lower bound, that is, there is $m$ such that $m \leq_{s} c$ for all $c$ in $C$.

Theorem 4.2. Let $X$ be a Banach space, $S$ an ordering cone and $C$ a nonempty subset of $X$. Assume that $S$ has a closed and bounded base $\Theta$. If either of the following is satisfied, then $S E(C, S)$ is norm-dense in the nonempty set $E(C, S)$ :

(1) $C$ is weakly compact;

(2) $C$ is weakly closed and S-lower bounded while $\Theta$ is weakly compact.

Proof. Since $S$ has a closed and bounded base, $S$ is closed. Moreover, it is Daniell [Borwein 3]. According to Theorem 2.1 in [Borwein 4], $E(C, S)$ is 
then nonempty if either $C$ is weakly compact or $C$ is (weakly) closed and $S$-lower bounded. Let $x_{0}$ be in $E(C, S)$. Then

$$
\left(C-x_{0}\right) \cap-S=\{0\} .
$$

Since $S$ has a base $\Theta$, for all $\delta>0$, one has

$$
\left(C-x_{0}\right) \cap-\delta \Theta=\varnothing .
$$

We claim that for any $\delta>0$, there is an $\varepsilon>0$ such that

$$
\left(C-x_{0}\right) \cap-S_{\varepsilon} \subset \delta B .
$$

Indeed, if (4.5) were false for some $\delta>0$, we would be able to find $c_{n}$ in $C$, $\theta_{n}$ in $\Theta, b_{n}$ in $B$ and $\lambda_{n}>0$ such that $\left\|c_{n}-x_{0}\right\|>\delta$ and

$$
c_{n}-x_{0}=-\lambda_{n}\left(\theta_{n}+(1 / n) b_{n}\right) \text {. }
$$

Without loss of generality, we may assume $\lambda_{n}$ converges. We consider two cases separately.

(1) When $C$ is weakly compact, $\left(c_{n}\right) \subset C$ is bounded. Extracting subsequences if necessary we may assume that $c_{n}$ converges weakly to some $c$ in $C$. If $\lambda_{n} \rightarrow \infty$, then by (4.6) we have

$$
-\theta_{n}=\lambda_{n}^{-1}\left(c_{n}-x_{0}\right)+(1 / n) b_{n} \rightarrow 0 .
$$

This is impossible because $0 \notin \mathrm{cl}(\Theta)$. Thus $\lambda_{n} \rightarrow \lambda<\infty$. It is clear that $\lambda>0$ as $\left\|c_{n}-x_{0}\right\|>\delta$. Hence

$$
\mathrm{w}-\lim \theta_{n}=\lambda^{-1}\left(x_{0}-c\right)
$$

is in $\Theta$ because $\Theta$ is weakly closed. Thus

$$
\left(C-x_{0}\right) \cap-S \neq\{0\} .
$$

This contradicts (4.3). Therefore (4.5) holds.

(2) Suppose now that $C$ is weakly closed and $S$-lower bounded, and $\Theta$ is weakly compact. Then by the weak-compactness of $\Theta$, we may assume that $\theta_{n}$ converges weakly to some $\theta_{0}$ in $\Theta$. If $\lambda_{n} \rightarrow \infty$, then

$$
\lambda_{n}^{-1}\left(c_{n}-x_{0}\right) \rightarrow-\theta_{0} \in-S .
$$

Since $\lambda_{n}^{-1} \rightarrow 0$ and $c_{n}$ are in $C, 0 \neq-\theta_{0}$ is in $R_{\sigma}(C) \cap-S$. This is a contradiction because $C$ is assumed to be $S$-lower bounded and hence

$$
R_{\sigma}(C) \cap-S=\{0\} .
$$

Thus, $\lambda_{n} \rightarrow \lambda<\infty$. Since $\Theta$ is bounded and $\left\|c_{n}-x_{0}\right\|>\delta$, (4.6) shows $\lambda>0$. Now $c_{n}$ converge weakly to $c:=x_{0}-\lambda \theta_{0}$. As $C$ is weakly closed, $c$ is in $C$. But $c-x_{0}=-\lambda \theta_{0} \in-S \backslash\{0\}$ contradicts the fact that $x_{0}$ is in $E(C, S)$. Thus (4.5) holds in this case too.

Note (4.5) implies that for small $\varepsilon$ the set $C_{\varepsilon}:=C \cap\left(x_{0}-S_{\varepsilon}\right)$ has arbitrarily small diameter. In each case $S_{\varepsilon}$ is Daniell as $S$ has a bounded closed base (Theorem 1.1(5)), and so Theorem 2.1 in [Borwein 4] applies. Thus $E\left(C_{\varepsilon}, S_{\varepsilon}\right)$ is nonempty. Clearly any point in $E\left(C_{\varepsilon}, S_{\varepsilon}\right)$ is actually in $E\left(C, S_{\varepsilon}\right)$, and so lies in $S E(C, S)$, by Corollary 2.6. Finally, let $y \in C_{\varepsilon} \cap E\left(C, S_{\varepsilon}\right)$, then $y$ belonging to $C_{\varepsilon}=C \cap\left(x_{0}-S_{\varepsilon}\right)$ implies that $y-x_{0} \in\left(C-x_{0}\right) \cap-S_{\varepsilon}$. Hence $\left\|y-x_{0}\right\|<\delta$, and we conclude that there is a point in $S E(C, S)$ arbitrarily close 
to $x_{0}$. As $x_{0}$ was arbitrarily chosen from $E(C, S)$, we see that $S E(C, S)$ is dense in $E(C, S)$.

Observe that the theorem can be viewed as giving density results for Borwein efficiency, Henig efficiency and Hartley efficiency (as super efficient points are in appropriate settings efficient in the sense of those authors). Even these specializations extend many density results in the literature.

\section{ChebysheV SCALARIZATIONS}

It is a fundamental principle in vector optimization that optimal elements can be characterized as optimal solutions of certain scalar optimization problems. We have seen that in some important special cases super efficiency can be satisfactorily characterized by scalar optimization problems. In the following we characterize super efficiency by Chebyshev scalarizations, i.e. characterize super efficiency in terms of certain equivalent norms on $X$.

Definition 5.1. We say that a norm $\|\cdot\|$ is $S$-monotone if

$$
0 \leq_{S} x \leq_{S} y \text { implies that }\|x\| \leq\|y\| \text {. }
$$

In a normed linear space $X$, when the ordering cone $S$ is normal and has nonempty interior and a set $C$ is contained in the interior of $S$, then each efficient point in $C$ can be expressed as those points in $C$ with least norm for the equivalent $S$-monotone norm defined by

$$
\||x|\|:=\inf \left\{t>0 \mid x \in t\left[-x_{0}, x_{0}\right]_{S}\right\},
$$

where $x_{0} \in E(C, S)$. First, we prove a lemma which shows that the norm defined by $(5.1)$ is indeed a norm equivalent to the original norm $\|\cdot\|$ of the space.

Lemma 5.2. Let $X$ be an ordered normed space with the norm $\|\cdot\|$. Suppose that the ordering cone $S$ has nonempty interior and is normal. If $x_{0}$ is in $\operatorname{int}(S)$, then the norm defined by (5.1) is $S$-monotone and equivalent to the norm $\|\cdot\|$. Proof. The proof that $\|\cdot\| \|$ defined by (5.1) is a norm can be found in [Jahn 1, p. 27]; that

$$
x^{\prime} \in[0, x]_{S} \Rightarrow\left\|\left|x^{\prime}\||\leq\||x|\|\right.\right.
$$

is easy and can be seen in [Jahn 1, p. 112]. We show \|\|$\cdot \| \mid$ is an equivalent norm. Now as $\operatorname{int}(S) \neq \varnothing, \operatorname{int}\left(\left[-x_{0}, x_{0}\right]_{S}\right) \neq \varnothing$. We may assume that $\alpha B \subset$ $\left[-x_{0}, x_{0}\right]_{S}$, where $B$ is the closed unit ball in $X$. Let $0 \neq x$ be in $X$, then $x\|x\|^{-1}$ is in $B$ and hence $\alpha x\|x\|^{-1} \in\left[-x_{0}, x_{0}\right]_{S}$. By the definition of \|\|$\cdot\|\|$, $\alpha\|\| x\|\mid\| x \| \leq 1$. Hence, $\left\|\left|x\left\|\mid \leq \alpha^{-1}\right\| x \|\right.\right.$. The fact that for some $k>0$ $\|x\| \leq k\||x \||$ follows from the normality of $S$. Hence, $\| \mid \cdot\|\|$ is an equivalent norm.

Proposition 5.3. Let the ordering cone $S$ be normal with nonempty interior. If the set $C$ is contained in $\operatorname{int}(S)$ and $x_{0}$ is in $E(C, S)$, then there exists an equivalent $S$-monotone norm $\||\cdot| \mid$ with

$$
1=\left\|\left|x_{0}\right|\right\|=\min \{|||x| \||| x \in C\} .
$$

Proof. Let

$$
\||x|\|:=\inf \left\{t>0 \mid x \in t\left[-x_{0}, x_{0}\right]_{s}\right\}
$$


By Lemma 5.2, $\|\cdot \cdot\|$ is a monotone norm which is equivalent to $\|\cdot\|$ with $\left\|\left|x_{0} \|\right| \leq 1\right.$. Suppose $\||x| \|<1$ for some $x$ in $C$. Then $x$ lies in $t\left[-x_{0}, x_{0}\right]_{S}$ for some $0<t<1$ and so $x \leq_{S} x_{0}, x \neq x_{0}$. But $\left(x_{0}-S\right) \cap C=\left\{x_{0}\right\}$ because $x_{0} \in E(C, S)$. Hence (5.2) holds.

Lemma 5.4. In an ordered Banach space whose ordering cone $S$ has a bounded base, there is an equivalent norm defined by

$$
\||x|\|:=\inf \left\{t>0 \mid x \in t[-a, a]_{S_{\varepsilon}}\right\}
$$

(for any $0 \neq a$ in $S$ and fixed $\varepsilon>0$ chosen sufficiently small) satisfies with the property that for some $\delta>0$,

$$
0 \leq_{s} x \leq_{s} y \Rightarrow\|\| y\|\| \geq\|x\|\|+\delta\|\|y-x\| \| .
$$

Proof. Let $\phi$ be in $S^{+i}$ such that $\phi(s) \geq\|s\|$ for all $s$ in $S$, as is possible since $S$ has a bounded base. Let $\Theta^{*}:=\phi^{-1}(1) \cap S$. Then $\Theta^{*}$ is a bounded base for $S$. Pick $\varepsilon>0$ small enough that $\Theta_{\varepsilon}^{*}$ is a base. The norm defined by (5.4) is indeed an equivalent $S_{\varepsilon}$-monotone norm by Lemma 5.2 because $S_{\varepsilon}\left(\Theta^{*}\right)$ is normal and has $a$ in its interior. Thus, there are $\beta>0, \beta_{1}>0$ such that

$$
\beta_{1}\|x\| \geq\|x\|\|\geq\| x \| \text {. }
$$

Suppose $0 \leq_{s} \leq_{s} y$ with $y \neq x$. Let $s_{0}:=y-x$. Then $s_{0}$ is in $S /\{0\}$. Set $x^{*}:=x / \phi\left(s_{0}\right), y^{*}:=y / \phi\left(s_{0}\right), s_{0}^{*}:=s_{0} / \phi\left(s_{0}\right)$. Then $s_{0}^{*} \in \Theta^{*}$. Now

$$
s_{0}^{*}+\varepsilon B \subset \varepsilon B+\Theta^{*} \subset S_{\varepsilon} \text {. }
$$

Thus, $s_{0}^{*}-\varepsilon y^{*} /\left\|y^{*}\right\| \in S_{\varepsilon}$ and

$$
\left(\left\|y^{*}\right\|-\varepsilon\right) y^{*} /\left\|y^{*}\right\|=x^{*}+s_{0}^{*}-\varepsilon y^{*} /\left\|y^{*}\right\| \geq_{s_{\varepsilon}} x^{*} \geq_{s} 0 .
$$

In particular, $\left\|y^{*}\right\| \geq \varepsilon$ since $y^{*} \in S_{\varepsilon}$ and $S_{\varepsilon}$ is pointed while $y$ is nonzero. As $\||\cdot|\|$ is $S_{\varepsilon}$-monotone, $\left(\left\|y^{*}\right\|-\varepsilon\right)\left\||| y^{*}\left|\|/\| y^{*}\|\geq\| x^{*}\right|\right\|$ and

$$
||\left|y^{*}\right||| \geq||\left|x^{*}\right||+\varepsilon||| y^{*}||\left|/\left\|y^{*}\right\| \geq\right||| x^{*}|| \mid+\varepsilon \beta .
$$

Therefore,

$$
\begin{aligned}
\|y\| & \geq\||| x|\|+\varepsilon \beta \phi(y-x) \geq\|||x|\|+\varepsilon \beta\|y-x\| \\
& \geq\left\|| | x \left|\left\|+\varepsilon \beta \beta_{1}^{-1}|\|y-x\||=\right\| x\||+\delta\|\mid\| y-x\|\|\right.\right.
\end{aligned}
$$

where $\delta:=\varepsilon \beta \beta_{1}^{-1}$. If $y=x$ this also holds and (5.5) is satisfied.

We observe that we could have used any equivalent $S_{\varepsilon}\left(\Theta^{*}\right)$-monotone norm.

Let us say that a norm satisfying (5.5) is strongly $S$-monotone. Clearly any such norm is strictly $S$-monotone in the sense that

$$
0 \leq_{s} x \leq_{s} y \text { and } x \neq y \text { implies that }\|x\|<\|y\| \text {. }
$$

Now we are able to give a characterization of $x_{0}$ being super efficient in terms of Chebyshev scalar optimization.

Theorem 5.5. In an ordered Banach space, if the closed ordering cone $S$ has a bounded base $\Theta$ and $C$ is contained in $S \backslash\{0\}$, then the following are equivalent:

(i) $x_{0} \in S E(C, S)$;

(ii) There is an equivalent strongly $S$-monotone norm ||$|\cdot|||$ such that

$$
\left\|\mid x_{0}\right\| \|=\min \{|||x| \||| x \in C\} .
$$


Proof. Let $x_{0} \in S E(C, S)$. Consider $\Theta$ as built in Lemma 5.4. Select a sufficiently small $\varepsilon>0$ so that

(i) the norm defined in Lemma 5.4 with $a:=x_{0}$ is strongly $S$-monotone and

(ii) $x_{0} \in E\left(C, S_{\varepsilon}(\Theta)\right)$; since $x_{0}$ lies in int $S_{\varepsilon}$. As in the proof of Proposition 5.3 we see that $(5.6)$ holds.

Conversely, suppose there exists an equivalent strongly $S$-monotone norm such that (5.6) is satisfied. Suppose that $y$ is in $X$ with $c-x_{0} \leq_{s} y$ for some $c$ in $C$. Then $0 \leq_{s} c \leq_{s} x_{0}+y$. This implies that

$$
\left\||| c||+\delta||\left|x_{0}+y-c\right||| \leq||\left|x_{0}+y \|\right| \mid .\right.
$$

Hence, using (5.6),

$$
\left\||| x_{0}|||+\delta||| x_{0}+y-c|||\leq||| x_{0}|||+||y|\right\| \mid
$$

which, in turn, implies that

$$
\delta\left\||| x_{0}-c|\|-\delta\|||y|\right\| \leq\||| y \mid\| .
$$

Therefore, $\left\|\left|x_{0}-c\right|\right\| \leq\left(1+\delta^{-1}\right)\|y\| \|$, i.e. $x_{0} \in S E(C, S)$.

Note that when the cone $S$ has a weakly compact base, the base $\Theta$ constructed above is also weakly compact (any other closed bounded base is). In addition, super efficient and proper efficient points coincide (by Proposition 3.7) and so we have the following corollary which extends a result in [Jahn 2].

Corollary 5.6. In an ordered Banach space, if the closed ordering cone $S$ has a weakly compact base $\Theta$ and $C$ is contained in $S \backslash\{0\}$, then the following are equivalent:

(i) $x_{0} \in P E(C, S)$;

(ii) There is an equivalent strongly $S$-monotone norm $\||\cdot| \mid$ such that

$$
||\left|x_{0}\right| \|=\min \{|||x||| \mid x \in C\} .
$$

\section{Conclusion}

It should be clear that super efficiency is a very flexible kind of proper efficiency and that there is a lot more that can be said about this concept. Some other results about super efficiency will be summarized in forthcoming papers. Simple expressions of super efficiency in vector lattices and scalarization results of super efficiency provide promises of practical application of the theory. Density results of super efficiency can be applied to establish some tangency formulas in nonsmooth analysis as demonstrated in [Zhuang 1]. We hope that super efficiency will enter the vector optimization literature and find its application in optimization practice.

\section{REFERENCES}

[Benson 1] H. P. Benson, An improved definition of proper efficiency for vector maximization with respect to cones, J. Math. Anal. Appl. 71 (1979), 232-241.

[Borwein 1] J. M. Borwein, Proper efficient points for maximizations with respect to cones, SIAM J. Control Optim. 15 (1977), 57-63.

[Borwein 2] _ The geometry of Pareto optimality, Math. Oper. Statist. 11 (1980), 235-248.

[Borwein 3] Continuity and differentiability of convex operators, Proc. London Math. Soc. 44 (1982), 420-444. 
[Borwein 4] _ On the existence of Pareto efficient points, Math. Oper. Res. 9 (1983), 64-73.

[Borwein 5] _ Norm duality for convex processes and applications, J. Optim. Theory Appl. 48 (1986) 22-29.

[Borwein 6] _ Convex cones, minimality notions and consequences, Recent Advances and Historical Development of Vector Optimization (J. Jahn and W. Krabs, eds.), Springer-Verlag, New York, 1987, pp. 64-73.

[Dauer-Stadler] J. P. Dauer and W. Stadler, $A$ survey of vector optimization in infinite dimensional spaces, Part II, J. Optim. Theory Appl. 51 (1986), 205-241.

[Dauer-Saleh] J. P. Dauer and O. A. Saleh, A characterization of proper minimal points as solutions of sublinear optimization problems (to appear).

[Geoffrion 1] A. M. Geoffrion, Proper efficiency and the theory of vector maximization, J. Math. Anal. Appl. 22 (1968), 618-630.

[Hartley 1] R. Hartley, On cone-efficiency, cone-convexity and cone-compactness, SIAM J. Appl. Math. 34 (1978), 211-222.

[Henig 1] M. I. Henig, Proper efficiency with respect to cones, J. Optim. Theory Appl. 36 (1982), 387-407.

[Holmes 1] R. B. Holmes, Geometric functional analysis and its applications, Springer-Verlag, New York, 1975.

[Hurwicz 1] L. Hurwicz, Programming in linear spaces, Studies in Linear and Nonlinear Programming, (K. Arrow, L. Hurwicz and H. Uzawa, eds.), Stanford Univ. Press, Standford, Calif., 1958, pp. 38-102.

[Jameson 1] G. Jameson, Ordered linear spaces, Lecture Notes in Math., vol. 141, SpringerVerlag, Berlin, 1970.

[Jahn 1] J. Jahn, Mathematical vector optimization in partially ordered linear spaces, Verlag Peter Lang, Frankfurt am Main, 1986.

[Jahn 2] Scalarization in vector optimization, Math. Programming 29 (1984), 203-218.

[Jahn 3] _ A generalization of a theorem of Arrow, Barankin and Blackwell, SIAM J. Control Optim. 26 (1988), 999-1005.

[Klee 1] V. L. Klee, Separation properties of convex cones, Proc. Amer. Math. Soc. 6 (1955), 313-318.

[Kuhn-Tucker] H. W. Kuhn and A. W. Tucker, Nonlinear programming, Proceedings of the Second Berkeley Symposium on Mathematical Statistics and Probability, (J. Neyman, ed.), University of California Press, Berkeley, 1951, pp. 481-492.

[Peressini 1] A. L. Peressini, Ordered topological vector spaces, Harper \& Row, New York, 1967.

[Zhuang 1] D. M. Zhuang, Regularity and minimality properties of set-valued structures in optimization, Ph.D Dissertation, Dalhousie Univ., 1989.

Department of Combinatorics and Optimization, University of Waterloo, Waterloo, Ontario, Canada N2L 3G1

E-mail address: jmborwei@orion.uwaterloo.ca

Department of Mathematics, Mount St. Vincent University, Halifax, Nova Scotia, CANADA B3M 2J6

E-mail address: zhuangd@ash.msvu.ca 